\title{
Avaliação do Nível de Ruído Causado por Diferentes Conjuntos Mecanizados
}

Luiz Henrique de Souza' Haroldo Carlos Fernandes ${ }^{2}$ Edney Leandro da Vitória ${ }^{3}$

\author{
Evaluation of Noise Levels Caused by \\ Different Groups of Mechanical \\ Equipment
}

'Engenheiro Agrícola, Mestrando em Engenharia Agrícola do Departamento de Engenharia Agrícola da Universidade Federal de Viçosa-MG

${ }^{2}$ Professor Adjunto do Departamento de Engenharia Agrícola da Universidade Federal de Viçosa - MG. ${ }^{3}$ Professor Substituto do centro Agropecuário da Universidade Federal do Espírito Santo - ES.
O presente estudo busca avaliar e comparar os níveis de ruído durante uma operação agrícola, desde a fase de aragem do terreno, até a colheita, para que, assim, em situação real de trabalho no campo, sejam feitas as devidas análises dos riscos a que estão sujeitos os operadores de máquinas, durante todo o manejo de uma cultura. Para realização dos ensaios, foram utilizados: trator $(73 \mathrm{cv})$, arado e grade de discos, aplicador de calcáreo, pulverizador e colhedora, onde foram feitos testes com o trator, tracionando cada um dos implementos, em velocidade e operações diferentes, com o motor mantido na rotação de $1700 \mathrm{rpm}$. Para a colhedora, foram utilizadas, também, velocidades de avanço diferentes, avaliando-se o nível de ruído à esquerda e à direita do operador. Em relação às médias dos níveis de ruído, os valores se encontraram muito elevados, ultrapassando os limites estipulados, gerando grande desconforto ao operador. A colhedora apresentou maior desconforto ao operador, não apresentando diferença significativa entre a mesma, parada ov em movimento. Mesmo estando o trator e/ou colhedora parados, os mesmos apresentaram níveis de ruído bem superior, ao mínimo estipulado apresentando assim, risco não só a quem opera, como também a quem está próximo dos mesmos.

Palavras-chaves Nível de Ruído, Máquinas Agrícolas.

This study endeavors efforts were therefore made to evaluate and compare the noise levels generated during agricultural operations, ranging from the plowing stage through to the harvest, so that the appropriate to be therefore able to conduct the risk analyses be made in real-life field conditions to determine exactly what machine operators are subjected to during an entire cultivation process. The machinery used in the tests consisted of: a $73 \mathrm{hp}$ tractor, a plow, disk grader, artificial manure and lime spreader, crop sprayer and harvester. The tests were conducted with the tractor towing each implement at varying speeds and in different operations with the engine running at $1700 \mathrm{rpm}$. The harvester was also operated using different forward gear speeds. The noise levels recorded on both the operator's right and lefthand sides were very high in relation to the average noise levels and exceeded the stipulated limits, causing much operator discomfort. The harvester generated the most operator discomfort, with little difference between when in motion and when not in motion. Both the tractor and the harvester when not in motion generated noise levels far higher than the stipulated minimum levels, presenting thereby a risk not only to the operator but also to whoever was close by.

Keywords Noise Level, Agricultural Machinery. 


\section{Introdução}

Dentre muitos fatores ambientais que prejudicam o trabalhador, o ruído pode ser considerado um dos principais, pois pode causar danos auditivos, assim como, outras conseqüências. Infelizmente, o setor agrícola observou esse problema tardiamente, tanto que, na área rural, pouco, ou quase nada, existe a respeito do ruído em máquinas agrícolas e, conseqüentemente, não se pode tirar nenhuma conclusão sobre os problemas ocupacionais, em trabalhadores agrícolas. De maneira geral, o trator se coloca em destaque, quando se pesquisa a relação homem-máquina, na área rural. Qualquer estudo, por mais superficial que seja, demonstra que a frota nacional de tratores não está projetada, de acordo com as necessidades do trabaIhador, mas sim, com as do trabalho. $O$ tratorista tem que, ao mesmo tempo, controlar o implemento e manter o trator alinhado, enquanto é submetido, durante horas, ao sol, à chuva, ao frio, à poeira e à fumaça do escapamento, além de um nível de ruído e de vibrações desumanos. No ano de 1897, na Suécia, foram realizados os primeiros ensaios oficiais com máquinas agrícolas, porém, o teste para tratores da Universidade de Nebraska é o mais famoso e conhecido, mundialmente. Os "Testes de Nebraska" servem de base para todos os ensaios de hoje. Eles se baseiam em 2 tipos de testes: ensaio na tomada de potência e ensaio na barra de tração (SILVEIRA, 1978). Até então, não existia nenhuma preocupação com o nível de ruído em tratores. A primeira Legislação que estabelecia valores máximos para - ruído em veículos e máquinas surgiu, na Alemanha em 1958. Foi criada uma tabela que impunha valores decrescentes para o ruído, em veículos, a cada ano de fabricação. Com a publicação da RECOMENDAÇÃO ISO R 362, a legislação passou a considerar, como limite máximo de ruído, para máquinas agrícolas, a faixa de 85 a 89 dBA (BURK, 1969). Em 1959, surgiu a norma para ensaio de tratores da Organização de Cooperação e Desenvolvimento Econômico (OCDE). Na sua versão de 1970, ela divide os ensaios com tratores em 2 tipos: 8 ensaios obrigatórios e 5 ensaios facultativos. Para os ensaios obrigatórios, 2 deles se referem ao ruído produzido por tratores: "ruído produzido pelo trator, no meio ambiente" e "medida do ruído no ouvido do tratorista" (FERNANDES, 1991). Segundo FERNANDES (1991), a maioria dos países da Europa adotam as normas da OCDE, como referência. Como exemplo, citam-se a França, que adotou todos os ensaios (inclusive os de ruído), sob a coordenação do Centro Nacional de Estudo e de Experimentação de Máquinas Agrícolas (CNEEMA); e a Inglaterra, que normalizou os ensaios de tratores, conforme as normas BS 1794 e BS 2800, e, para ensaios específicos de ruído em veículos, usa as normas BS 3489 - 1962 - "Sound Level Meters" e BS 3534 - 1962 - "Sound Level Meters for the Measurement of Noise Emitted by Motor Vehicles". Nos Estados Unidos, várias normas disciplinam o nível de ruído em máquinas, sendo as principais, segundo FERNANDES (1991): SAE - J919A - 1971 - "SAE Recomended Pratice, Sound Measurement at the Operator Station for Agricultural and Construction Equipament" e "SAE - J1013 - 1973 "ASAE Recomended Pratice, Measurement of Whole Body Vibration of the seated operator of Agricultural Equipament". No Brasil, a Associação Brasileira de Normas Técnicas (ABNT) possui inúmeras normas a respeito de medições de ruído em máquinas, sendo as principais para máquinas agrícolas: NBR - 9999 (ASSOCIAÇÃO ... - NBR, 1987) - "Medição do Nível de Ruído, no Posto de Operação, de Tratores e Máquinas Agrícolas" e NBR - 10400 (NBR, 1988) - "Tratores Agrícolas - Determinação das Características Técnicas e Desempenho". Há controvérsia muito grande a respeito do limite de ruído ocupacional. Vários estados americanos fixaram o limite em $90 \mathrm{dBA}$. $O$ critério das leis federais americanas (NIOSH - "National Institute for Occupational Safety and Health") também fixou $90 \mathrm{dBA}$, enquanto várias pesquisas científicas têm evidenciado ser este nível elevado. Normas de alguns países já reduziram esse limite para 85 ou $80 \mathrm{dBA}$. Estudando o risco auditivo de trabalhadores expostos a diversos níveis de ruído, MERLUZZI et al. (1987) afirmaram que "exposição a ruído ocupacional de $85 \mathrm{dBA}$ pode modificar o limiar auditivo de certa porcen- 
tagem da população exposta ao ruído".

Na tabela 1, mostram-se os limites de exposição ocupacional ao ruído, confor- me normas nacionais de diversos países (FERNADES, 1991).

Tabela 1 Limite de exposição ocupacional ao ruído, conforme as normas nacionais de diversos países.

\begin{tabular}{ccccc}
\hline País & $\begin{array}{c}\text { Nível de } \\
\text { ruído }\end{array}$ & $\begin{array}{c}\text { Tempo de } \\
\text { exposição } \\
(h)^{*}\end{array}$ & $\begin{array}{c}\text { Nível } \\
\text { máximo }\end{array}$ & $\begin{array}{c}\text { Nível de rúdo } \\
\text { de impacto } \\
(\mathrm{dB})\end{array}$ \\
\hline Alemanho & 90 & 8 & $\cdot$ & - \\
Japão & 90 & 8 & $\cdot$ & - \\
França & 90 & 40 & - & - \\
Bélgico & 90 & 40 & 110 & 140 \\
Inglaterra & 90 & 8 & 135 & 150 \\
Itália & 90 & 8 & 115 & 140 \\
Dinamarco & 90 & 40 & 115 & - \\
Suécia & 85 & 40 & 15 & - \\
USA - OSHA & 90 & 8 & 115 & - \\
LSA - NIOSH & 85 & 8 & - & 140 \\
Canadó & 90 & 8 & 115 & - \\
Austrália & 90 & 8 & 115 & - \\
Holanda & 80 & 8 & 115 & - \\
Brasil & 85 & 8 & &
\end{tabular}

*Tempo de exposição diária ou semanal.

OSHA: "Occupational Safety and Health Administration".

NIOSH: "National Institute for Occupational Safety and Health".

\section{Objetivos}

1. Avaliar e comparar os níveis de ruído durante operação agrícola para os conjuntos trator-implemento e colhedora.

2. Comparar os resultados, com as normas vigentes no Brasil.

\section{Materiais e métodos}

Os ensaios foram conduzidos em uma área de aproximadamente 1 ha, pertencente ao Departamento de Engenharia Agrícola (DEA) da Universidade Federal de
Viçosa (UFV). A área está localizada no município de Viçosa, MG, com longitude de $42^{\circ} 52^{\prime} 40^{\prime \prime} \mathrm{W}$ (Gr) e latitude $20^{\circ}$ $45^{\prime} 20^{\prime \prime} \mathrm{S}$, a $658 \mathrm{~m}$ de altitude.

Nas operações agrícolas foram avaliados: Trator - $54 \mathrm{KW}-73 \mathrm{cv}-2000$ rpm; arado de discos reversível, marca CIPOLA, modelo "C3, 3 × 26", largura de corte de $1,10 \mathrm{~m}$ e peso aproximado de $4.905 \mathrm{~N}$; grade niveladora "off-set", marca BALDAN, $36 \times 17^{\prime \prime}$, largura de corte aproximada de $2,8 \mathrm{~m}$ e peso aproximado de $9.810 \mathrm{~N}$; distribuidor de calcáreo; pulverizador, bico Teejet $11003 \mathrm{vk}$; semeadora e colhedora auto-propelida. 
Antes da passagem das máquinas, foram coletadas 10 amostras em 3 profundidades, em pontos escolhidos aleatoriamente, na área onde foram feitos os testes. Utilizou-se um anel volumétrico, e o valor da densidade do solo foi obtida, dividindose a massa do solo, secada em estufa a $105^{\circ} \mathrm{C}$, pelo seu volume (EMPRESA... - EMBRAPA, 1979). Na área experimental, antes da passagem das máquinas, foram feitas leituras do índice de cone, em pontos distribuídos aleatoriamente, perfazendo-se um total de 10. As leituras foram efetuadas por meio de um penetrógrafo da marca SOIL CONTROL, modelo SC-60, com uma haste de $600 \mathrm{~mm}$ de comprimento e 9,53 mm de diâmetro, equipada com um cone de 129,3 $\mathrm{mm}^{2}$ de área de base, 12,83 mm de diâmetro $\mathrm{e}$ 30 graus de ângulo de vértice. $O$ índice de cone foi obtido através da média das leituras de 0 a $0,40 \mathrm{~m}$, em $\mathrm{kgf} / \mathrm{cm}^{2}$, transformada para unidade do Sistema Internacional de Medidas (MPa). Procurou-se, na obtenção das leituras, seguir as recomendações-padrão da ASAE S 313 (AMERICAN SOCIETY OF AGRICULTURAL ENGINEERS, 1990), que exige, para o teste, uma velocidade de penetração de $1.829 \mathrm{~mm}$ por minuto.

Para determinação do teor de água, utilizou-se o método-padrão de estufa, seguindo a metodologia empregada pela EMBRAPA (1979). As amostras foram coletadas na área, antes da passagem das máquinas, em 10 pontos, aleatoriamente, distribuídos na área, e em 3 diferentes profundidades.

O trator e colhedora foram avaliados nas condições em que se encontravam no campo, em trabalho. O local escolhido para realização dos testes era, relativamente plano, sem obstáculos e declives, os quais poderiam ocasionar deflexão do som. As medições não foram realizadas, com equipamento, em manobra, ou durante a aceleração e a desaceleração.

Antes do início de cada determinação dos níveis de ruído, mediram-se o ruído de fundo, a velocidade do vento, a umidade e a temperatura do ar. Embora tenha sido observada a condição climática favorável, em todas as medições, foi utilizado o protetor de ventos, no microfone do medidor de pressão sonora, com os intuitos de uniformizar as condições de leitura e evitar a influência de rajadas de vento que pudessem ocorrer.

A escala utilizada para medida do nível de ruído do trator e colhedora foi o decibel (dB). Os ruídos foram determinados em um medidor de pressão sonora (decibelímetro) da marca MINIPA, no circuito de resposta lenta (slow) e de equalização " $A$ ".

As determinações dos níveis de ruído foram baseadas na NBR 9999 (ABNT, 1987), que é uma norma específica para a medida de ruído, em tratores, e seus efeitos sobre o tratorista, em que são fixados todos os procedimentos de medidas. Embora as condições de operação tenham diferido daquelas prescritas pela norma, todos os procedimentos de medida, nela fixados, podem ser cumpridos, conforme realizado por FERNANDES (1991) e LIMA (1998).

O microfone do medidor foi posicionado na altura do ouvido do operador, conforme a norma NBR 9999 (ABNT, 1987).

Para a obtenção do ruído, gerado apenas pelo motor, foram feitas medições dos níveis de ruído, com o trator parado. Essa avaliação permitiu quantificar a contribuição do motor no ruído total do trator e determinar os níveis de ruído a que estão sujeitos os trabalhadores, próximos ao trator.

As leituras, um total de 10, foram realizadas próximas ao ouvido do operador, do lado onde estava posicionado o escapamento de saída dos gases do motor, onde se suponha ser o lado de maior nível de ruído, emitido pelos tratores.

Para avaliar a influência da força de tração requerida pelo implemento, realizaram-se ensaios, com variáveis, previamente fixadas: foram utilizados 1 trator, arado e grade de discos, aplicador de calcáreo, semeadora, pulverizador e colhedora. Realizaram-se os ensaios com o trator, tracionando cada um dos implementos, em velocidades e operações diferentes, o motor foi mantido na rotação de $1700 \mathrm{rpm}$. Para a colhedora, foram 
analisados velocidades e lados à esquerda e à direita do operador.

A medição dos níveis de ruído foi realizada, com o medidor posicionado próximo ao ouvido do operador durante a execução das operações, em uma área preestabelecida de $90 \mathrm{~m}^{2}$, sendo efetuadas 10 leituras, em períodos de 5 segundos, aproximadamente, caso a dispersão das leituras do nível de ruído obtida nas condições de medição excedesse a 3 dBA; outra medição era realizada até que a leitura de 3 medições sucessivas se limitassem a uma dispersão, na faixa de 3 dBA. As médias dessas leituras foram consideradas como resultado.

Na avaliação da influência da força de tração requerida, dos níveis de ruído durante uma operação agrícola, o experimento foi montado num delineamento fatorial $2 \times 2$, para velocidade do conjunto e profundidade de trabalho, respectivamente, com 10 repetições, para leituras dos níveis de ruído. Na avaliação da influência do lado de operação, para a colhedora, foi montado o experimento, num delineamento fatorial $2 \times 2$, para velocidade $\mathrm{e}$ lado de operação, respectivamente, conforme o número de repetições, citado acima.

\section{Resultados}

Os testes foram realizados, em uma área, onde os fatores que poderiam afetar a força de tração requerida; como declividade, altitude, condições de superfície do solo, umidade do solo, tipo de solo e resistência do solo à penetração, eram homogêneos, assegurando-se, assim, que as variações de velocidade e profundidade, para cada conjunto trator-implemento ensaiado, seriam suficientes, para uma variação, na força de tração.

Através da análise de variância, verificou-se que as interações, entre as velocidades e profundidades utilizadas, foram significativas a $5 \%$ de probabilidade, exceto nos conjuntos: trator-semeadora e trator-Aplicador de calcáreo. $\bigcirc$ coeficiente de variação apresentou valores entre 38 e $72 \%$, representando uma alta dispersão dos níveis de ruído, nas operações agrícolas consideradas.

\section{Trator com arado de discos}

Na tabela 2 podem ser vistos os resultados das médias dos níveis de ruído.

Tabela 2 Médias dos níveis de ruído (dBA), obtidas no trator com arado de discos, variando-se a velocidade e a profundidade da operação.

\begin{tabular}{c|c|c}
\hline \multirow{2}{*}{$\begin{array}{c}\text { Velocidade } \\
\left(\mathrm{Km} \cdot \mathrm{h}^{-1}\right)\end{array}$} & 0,18 & 0,25 \\
\cline { 2 - 3 } & $94,5 \mathrm{Aa}$ & $94,6 \mathrm{Aa}$ \\
4,8 & $96,5 \mathrm{Ab}$ & $97,6 \mathrm{Bb}$ \\
\hline
\end{tabular}

Médias seguidas pelas mesmas letras maiúsculas, na horizontal, e pelas mesmas letras minúsculas, na vertical, não diferem, entre si, a $5 \%$ de probabilidade, pelo teste de Tukey.

A maior média de nível de ruído foi en- contrada, quando se utilizaram a maior velocidade e a maior profundidade, indicando a tendência de elevação do nível de ruído, em função do aumento da força de tração requerida, obtida, na mesma rotação do motor do trator. 


\section{Trator com grade de discos}

Tabela 3 Médias dos níveis de ruído (dBA), obtidas no trator com grade de discos, variando-se a velocidade e o ângulo de travamento da grade.

\begin{tabular}{c|c|c}
\hline \multirow{2}{*}{$\begin{array}{c}\text { Velocidade } \\
\left(\mathrm{Km} \cdot \mathrm{h}^{-1}\right)\end{array}$} & \multicolumn{2}{|c}{ Profundidade } \\
\cline { 2 - 3 } & Maior Profundidade & Menor Profundidade \\
\hline 6,5 & $96,5 \mathrm{Aa}$ & $96,4 \mathrm{Aa}$ \\
8,0 & $97,2 \mathrm{Ab}$ & $95,8 \mathrm{Bb}$ \\
\hline
\end{tabular}

Médias seguidas pelas mesmas letras maiúsculas, na horizontal, e pelas mesmas letras minúsculas, na vertical, não diferem, entre si, a $5 \%$ de probabilidade, pelo teste de Tukey.

A maior média de nível de ruído foi encontrada, quando se utilizaram a maior velocidade e a maior profundidade, indicando a tendência de elevação do nível de ruído, em função do aumento da força de tração requerida, obtida, na mesma rota- ção do motor do trator.

\section{Trator com aplicador de calcáreo}

Os resultados das médias dos níveis de ruído para as velocidades foram analisadas, separadamente, já que não houve interação, entre essas duas variáveis, conforme análise de variância.

Tabela 4 Médias dos níveis de ruído (dBA), obtidas no trator, com aplicador de calcáreo para as velocidades utilizadas.

\begin{tabular}{c|c}
\hline Velocidade $\left(\mathrm{km} \cdot \mathrm{h}^{-1}\right)$ & Médias de ruído (dBA) \\
\hline 6.5 & $93,5 \mathrm{~A}$ \\
5.5 & $92,6 \mathrm{~A}$ \\
\hline
\end{tabular}

Médias seguidas pelas mesmas letras não diferem, entre si, a $5 \%$ de probabilidade, pelo teste de Tukey.

Embora não tenha ocorrido diferença significativa para as médias dos níveis de ruí- do para as duas velocidades estudadas, a maior média foi encontrada, quando se utilizou a maior velocidade, indicando a tendência de elevação do nível de ruído, em função do aumento da velocidade do trator.

\section{Trator com Semeadora}

Tabela 5 Médias dos níveis de ruído (dBA), obtidas do trator, com semeadora, variando-se a velocidade de trabalho.

Os resultados das médias dos níveis de ruído para as velocidades foram analisadas, separadamente, já que não houve interação entre essas duas variáveis, conforme análise de variância. 
Médias seguidas pelas mesmas letras não diferem entre si, a $5 \%$ de probabilidade, pelo teste de Tukey.

Não houve diferença significativa para as médias dos níveis de ruído, para as duas velocidades de trabalho estudadas para a mesma rotação do motor do trator.

\section{Trator com Pulverizador}

Tabela 6 Média dos níveis de ruído (dBA) obtidas do trator, com pulverizador, em função da velocidade e pressão utilizada.

\begin{tabular}{c|c}
\hline Velocidade $\left(\mathrm{km} \cdot \mathrm{h}^{-1}\right)$ & Médias de ruído $(\mathrm{dBA})$ \\
\hline 5,5 & $91,3 \mathrm{~A}$ \\
6,0 & $92,1 \mathrm{~B}$ \\
\hline
\end{tabular}

Médias seguidas pelas mesmas letras não diferem entre si, a $5 \%$ de probabilidade, pelo teste de Tukey.

A maior média do nível de ruído foi encontrada na maior velocidade, indicando a tendência de elevação do nível de ruído em função do aumento da velocidade, para o trator com pulverizador numa mesma pressão de trabalho.

\section{Colhedora}

Tabela 7 Média dos níveis de ruído (dBA) da colhedora, em função da velocidade e lado de operação.

\begin{tabular}{c|c|c}
\hline \multirow{2}{*}{$\begin{array}{c}\text { Velocidade } \\
\left(\mathrm{Km} \cdot \mathrm{h}^{-1}\right)\end{array}$} & Esquerdo & Direito \\
\hline 1,5 & $95,9 \mathrm{Aa}$ & $96,7 \mathrm{Ba}$ \\
0,8 & $96,8 \mathrm{Ab}$ & $97,0 \mathrm{Aa}$ \\
\hline
\end{tabular}

Médias seguidas pelas mesmas letras maiúsculas, na horizontal, e pelas mesmas letras minúsculas, na vertical, não diferem, entre si, a $5 \%$ de probabilidade, pelo teste de Tukey.

A maior média do nível de ruído foi encontrada, na menor velocidade, devido a uma maior trepidação da colhedora nesta situação e lado direito, já que o escapamento da mesma se situa deste lado.

\section{Nível de ruído com o trator parado}

Foi obtida uma média de nível de ruído de 89,6 dBA do lado do escapamento do mesmo.

\section{Nível de ruído com a semeadora parada}

Foi obtida uma média de nível de ruído de 96,8 dBA do lado do escapamento da colhedora.

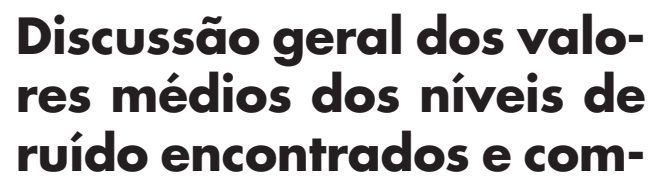




\section{paração direta com ou- tras publicações}

A tabela 8 mostra o tempo máximo, permi- tido de trabalho diário, para cada operação agrícola, considerando-se o maior nível de ruído médio encontrado para cada operação.

Tabela 8 Tempos de exposição diária permissivel às médias de ruído encontradas nas operações.

\begin{tabular}{c|c|c}
\hline Operação & $\begin{array}{c}\text { Níveis de ruído } \\
\text { médios encontrados } \\
(\mathrm{dBA})\end{array}$ & $\begin{array}{c}\text { Tempo máximo de } \\
\text { exposição } \\
\text { (minutos) }\end{array}$ \\
\hline Trator com arado de discos & 95,8 & 60 \\
Trator com grade de discos & 96,5 & 75 \\
Trator com aplicador de calcáreo & 93,0 & 60 \\
Trator com semeadora & 92,9 & 60 \\
Trator com pulverizador & 91,7 & 60 \\
Colhedora & 96,8 & 60 \\
Trator parado & 89,6 & 60 \\
Colhedora parada & 96,8 & 60 \\
\hline
\end{tabular}

\section{Conclusões}

Com relação às médias dos níveis de ruído encontradas nas medições, os valores se encontraram elevados, ultrapassando os limites estipulados pela Portaria 3214/78 - NR15 - CLT (cujo valor limite é de $85 \mathrm{dBA}$ para uma exposição máxima diária de 8 horas, sem protetores auriculares), os níveis geram grande desconforto ao operador.
A colhedora apresentou maior desconforto ao operador, não apresentando diferença significativa, entre a mesma, parada ou em operação.

Mesmo estando o trator e/ou colhedora parados, os mesmos apresentaram níveis de ruído, superiores ao mínimo estipulado, apresentando riscos a quem opera, e a quem está próximo dos mesmos.

\section{Referências Bibliográficas}

\author{
ABNT - ASSOCIAÇÃO BRASILEIRA DE \\ NORMAS TÉCNICAS - NBR 9999- Me- \\ dição do Nível de Ruído, no Pos-
}

to de Operação de Tratores e Máquinas Agrícolas. 1987.

$12 \mathrm{p}$. 
ABNT - ASSOCIAÇÃO BRASILEIRA DE NORMAS TÉCNICAS - NBR 10400Tratores Agrícolas - Determinação das Características Técnicas e Desempenho. 1988.

ABNT - ASSOCIAÇÃO BRASILEIRA DE NORMAS TÉCNICAS - NORMA NB - 95 - Níveis de ruído aceitáveis. 1966.

AMERICAN SOCIETY OF AGRICULTURAL ENGINEERS. ASAE Standards 1990. St. Joseph, Michigan, 1990. Não paginado.

BRASIL - Decreto-lei n. 5452 , de 1ำ de maio de 1943. Consolidação das leis trabalhistas, 1943.

BRASIL - Lei $\mathrm{n} .{ }^{\circ}$ 6514, de 22 de dezembro de 1977 - Altera o Capítulo V do Título II da Consolidação das Leis TrabaIhistas, relativo a segurança e medicina do trabalho, 1977.

EMBRAPA. Centro Nacional de Ciência do Solo. Manual de métodos de análise do solo. Rio de Janeiro, 1979. Não paginado.

FERNANDES, J. C. Avaliação dos níveis de ruído em tratores agrícolas e seus efeitos sobre o operador. Botucatu: UNESP, 1991. 192p. Tese (Doutorado em Energia na Agricultura) - Faculdade de Ciências Agronômicas - Universidade Estadual Paulista, 1991a.

FERNANDES, J. C.; SANTOS, J. E. G.; ANDREATTA, J. A. As fontes de ruído em tratores agrícolas - In: XX Congresso Brasileiro de Engenharia Agrícola, 1991 b Londrina-PR,. Anais... Sociedade Brasileira de Engenharia Agrícola, v.2, p. 1305-1316.

FERNANDES, J. C.; SANTOS, J. E. G.; ANDREATTA, J. A. Influência do implemento no ruído de operações agrícolas - In: XX Congresso Brasileiro de Engenharia Agrícola, 1991c, Londrina-PR. Anais... Sociedade Brasileira de Engenharia Agrícola, v. 2, p. 1327-1337.
FERNANDES, J. C; Estratégias para diminuição do ruído em tratores agrícolas Parte I: alteração da posição do sistema de exaustão - In : XXV Congresso Brasileiro de Engenharia Agrícola, 1996a, Bauru-SP. Anais... Sociedade Brasileira de Engenharia Agrícola. p. 172.

FERNANDES, J. C; Estratégias para diminuição do ruído em tratores agrícolas Parte II: Redimensionamento de posição do sistema de exaustão - ln : XXV Congresso Brasileiro de Engenharia Agrícola, 1996b, Bauru-SP. Anais... Sociedade Brasileira de Engenharia Agrícola. p.221.

FIEDLER, N. C. Avaliação ergonômica de máquinas utilizadas na colheita de madeira. Viçosa, MG, UFV. 1995. 126p. (tese M.S.).

FUNDACENTRO - Norma para avaliação da exposição ocupacional ao ruído Série Técnica de Avaliação de Riscos Ambientais - NHT-09 R/E - Revista Brasileira de Saúde Ocupacional, n. 14, 1986.

IIDA, I. Ergonomia: projeto e produção. São Paulo, Edgard Blucher, $1990.465 \mathrm{p}$.

IIDA, I; WIERZZBICKI, M. Ergonomia; notas de aulas. São Paulo, Faculdade de Engenharia Industrial, 1978. $299 p$.

ISO - INTERNATIONAL STANDARD ORGANIZATION - Norma ISO 1999

- Acoustics - Assessment of Occupational Noise Exposure for Hearing Conservation Purposes. 1975.

ISO - INTERNATIONAL STANDARD ORGANIZATION - Norma ISO 5131 - Acoustics - Tractors and Machinery for Agriculture and Forestry - Measurement of Noise at the Operator's Position. 1982. 8 p. 
LAVILLE, Ergonomia: engenharia humana. São Paulo: EPU/EDUSP, $1977.101 \mathrm{p}$.

LIMA, J. S. S.; FERNANDES, H. C.; VITÓRIA, E. L. Avaliação dos níveis de ruído de máquinas florestais em função do raio de afastamento. In: Congresso Brasileiro de Engenharia Agrícola, 1998, Poços de Caldas-MG. Anais... Sociedade Brasileira de Engenharia Agrícola, p. 70-72.

MASTRI, E., VIVI, A., FATTORINI, A., et al. Il rumore in alcune lavorazioni meccaniche agricole. In: CONGRESSO NAZIONALE DELLA SOCIETÀ

MEYER, R. E.; SCHWAB, C. V.; BERN, C. J. Tractor noise expousure levels for bean- bar riders. TRANSACTIONS of the

ASAE: v. 36(4) 1049-1056. 1993

MIALHE, L. G. Máquinas agrícolas: ensaios \& certificação. Piracicaba, São Paulo, FEALQ. 1996. $722 p$.

OLIVEIRA, N. V. ARAÚJO, M. C., BARBOSA, J. C. SOBRINHO, A. T. Investigação do nível de ruído a que o tratorista está exposto no preparo periódico do solo com trator de pneu. In: Congresso Brasileiro de Engenharia Agrícola, 1998, Poços de Caldas-MG. Anais... Sociedade Brasileira de Engenharia Agrícola, v. 3, p. 289-291. 